UDC 547.544.424.678.6

\title{
SYNTHESIS OF POLYIMIDES OF POLYCHLORINATED CYCLIC DICARBOXYLIC ACIDS ON THE BASIS OF DIENE-DIENOPHILIC BISIMIDES ON THE DIELS-ALDER REACTION
}

\author{
A.I.Alikhanova \\ Institute of Polymer Materials, NAS of Azerbaijan \\ aygun81@mail.ru
}

Received 17.07.2017

\begin{abstract}
The conditions for the synthesis of $\mathrm{N}$-aminoimides of polychlorinated cyclic dicarboxylic acids has been studied and the possibility to obtain hinge-ladder polyimides by the Diels-Alder reaction has been demonstrated.
\end{abstract}

Keywords: Diels-Alder reaction, bisimides, polyimides, acylation, bisimidodienes.

There exist many methods of producing of polyimides (PI) synthesis [1], however they are based mainly on a two-stage procedure [2]. Besides the hinged polyimides obtained by the known methods do not possess $100 \%$ imidization due to an incomplete conversion of polyamidoacidic links [1-3].

Polymerization by the Diels-Alder reaction is one of the promising and developing directions in the preparation of polyimides of various structures that promotes wide modification of their chemical composition and properties.

In this work the synthesis of polyimides with the using of $\mathrm{N}$-aminoimides of mixed diene-dienophiles of 1,2,3,4-tetrachlorcyclohexa1,3-diene-5,6- (V $a, b, c)$ and 2,3,4,5-tetrachlorobicyclo[4.4.0]deca-2,4-diene-8,9-dicarboxylic acids (VI $a, b, c)$ and maleic anhydride is described.

The acylation of $\mathrm{N}$-alkyl(aryl)aminoimides of 1,4,5,6,7,7-hexachlorobicyclo[2.2.1] hept-5-ene2,3-dicarboxylic acid (HCBCHDCA) (I $a, b, c)$ and 1,2,3,4,11,11-hexachlorotricyclo[6.2.1.0 $\left.0^{5,10}\right]$ undec-2-ene-7,8-dicarboxylic acid (HCTCUDCA) (II $a, b, c)$ with maleic anhydride in the environment of DMF at $150^{\circ} \mathrm{C}$ leads to obtaining N,N'maleinalkyl(aryl)substituted imides of 1,4,5,6,7,7hexachlorobicyclo[2.2.1] hept-5-ene-2,3-dicarboxylic acid (III $a, b, c)$ and endo, exo-1,2,3,4,11,11hexachlorotricyclo[6.2.1.0 $\left.0^{5,10}\right]$ undec-2-ene-7,8-dicarboxylic acid (IV $a, b, c$ ).

The obtained bisimides (III $a, b, c$ ) and (IV $a, b, c$ ) were brought into the reaction with a mixture of pyridine and acetic anhydride in DMF solution aiming at the preparation of diene-dienophile bisimides, N, N'-maleinalkyl(aryl)substituted imides of 1,2,3,4-tetrachlorocyclohexa-1,3diene-5,6-dicarboxylic acid (V $a, b, c)$ and endo, exo-2,3,4,5-tetrachlorobicyclo[4.4.0]deca-2,4-diene-8,9-dicarboxylic acid (VI $a, b, c)$. The reaction proceeded with heat evolution and temperature increase to $50^{\circ} \mathrm{C}$. Then the mixture was stirred again for $2 \mathrm{~h}$ at $110-120^{\circ} \mathrm{C}$.
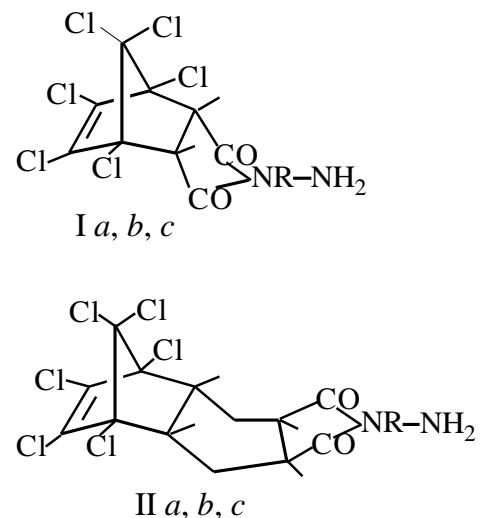

$\mathrm{R}=-\left(\mathrm{CH}_{2}\right)_{2},-\left(\mathrm{CH}_{2}\right)_{6},-\mathrm{Ph}$.

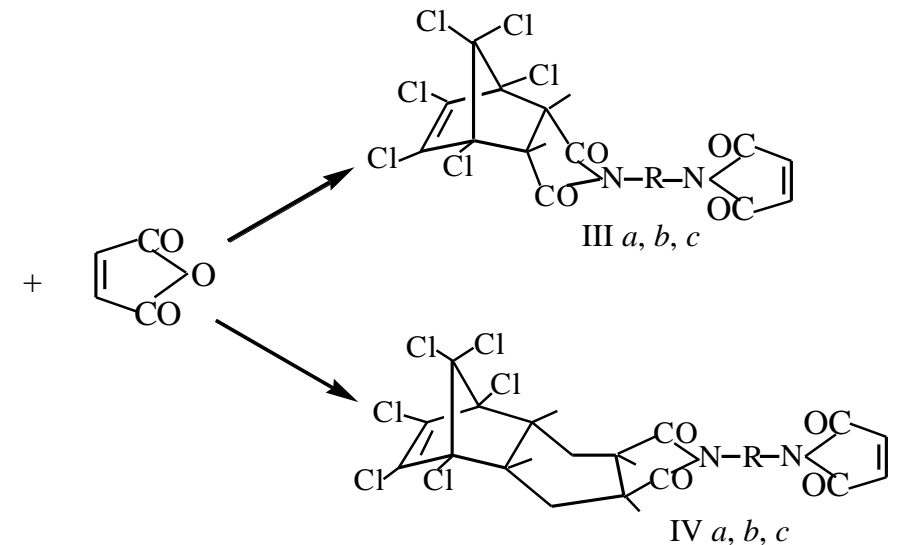

IV $a, b, c$ 


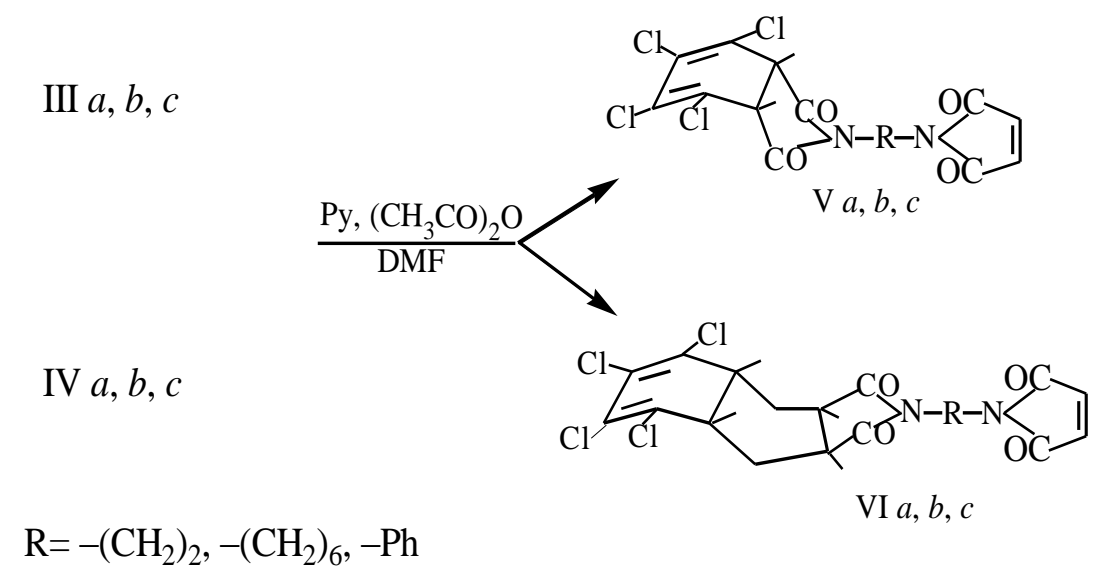

The structure of bisimidodienes ( $\mathrm{V} a, b, c)$ and (VI $a, b, c)$ has been proved by IR and UV spectroscopy. In the IR spectrum of bisimidodienes (V $a, b, c)$ and (VI $a, b, c)$ there are observed the characteristic absorption bands in the field of $1710-1780 \mathrm{~cm}^{-1}$ for the imide cycles, 1600 $1610 \mathrm{~cm}^{-1}$ for the $\mathrm{C}=\mathrm{C}, 750 \mathrm{~cm}^{-1}$ corresponding for the $\mathrm{C}-\mathrm{Cl}$ bonds vibrations (Figure 1).

The UV spectrum of bisimides ( $\mathrm{V} a, b, c)$ and (VI $a, b$ ), c contained the absorption bands characteristic of a diene system in the region $285 \mathrm{~nm}$. The presence of the diene system in bisimides of diene-dienophiles ( $\mathrm{V} a, b, c)$ and (VI $a, b, c$ ) was confirmed by their polycondensation in the presence of hydroquinone for 4-6 $\mathrm{h}$ at $150^{\circ} \mathrm{C}$.

In the process of polycondensation by the Diels-Alder the choice of the solvent in solution plays a decisive role $[5,6]$. We have studied seve- ral aprotic solvents (DMF, DMA and DMSO). The reaction was carried out at $150^{\circ} \mathrm{C}$ and a duration of 6-8 hours. It was found that with increasing in the solvent polarity the viscosity and the yield of polyimide is increased. The greatest yield of polymer is reared when using DMA as solvent. It reaches 90-95\%.

To determine the optimal conditions for the synthesis of polyimides, the influence of the concentration of the initial monomer, the temperature, and the duration of the reaction on the course of polycondensation of diene-dienophilic bisimides (V $a, b, c)$ and (VI $a, b, c$ ) has been studied.

In the IR spectra of polyimides VII and VIII there are the characteristic absorption bands the field of $1712,1780 \mathrm{~cm}^{-1}$ for the $\mathrm{C}=\mathrm{O}$, $600-750 \mathrm{~cm}^{-1}$ corresponding for the $\mathrm{C}-\mathrm{Cl}$ bonds vibrations (Figure 2).

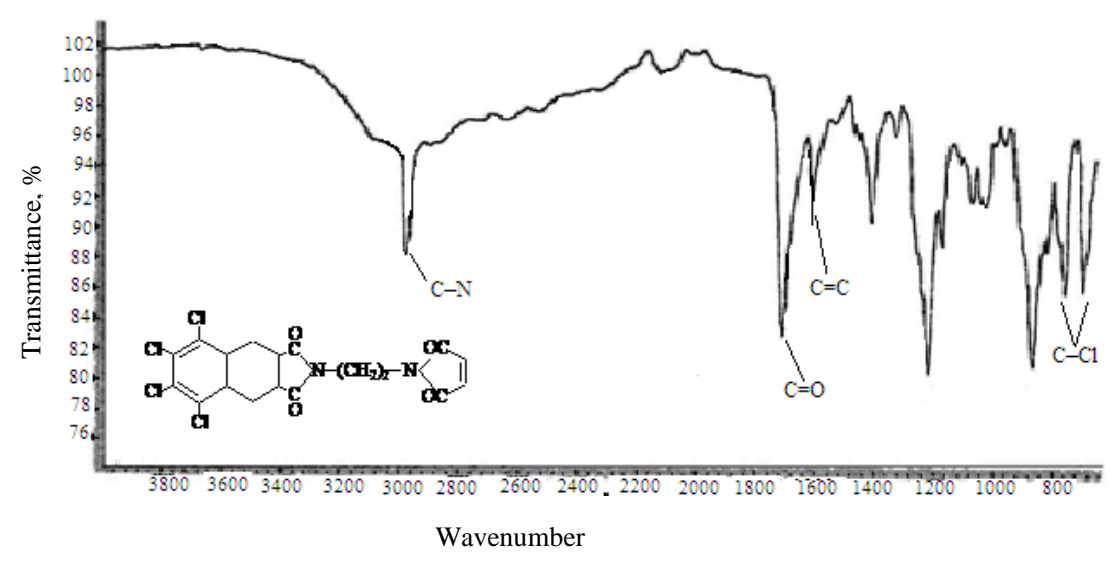

Fig. 1. IR spectrum of bisdiene VI $a$. 
(N)

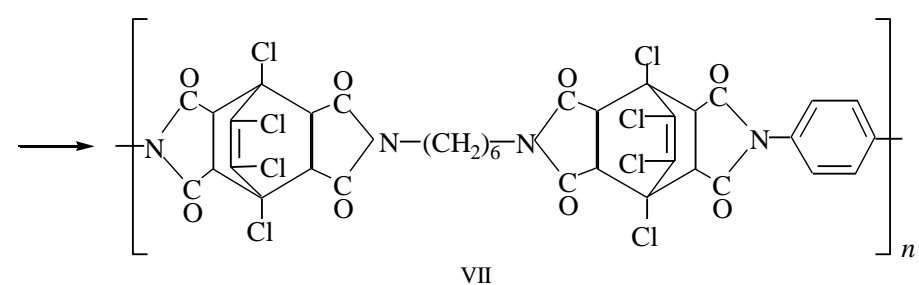

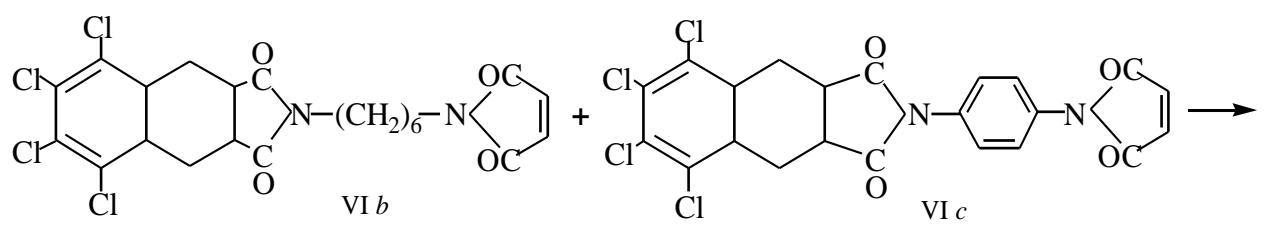

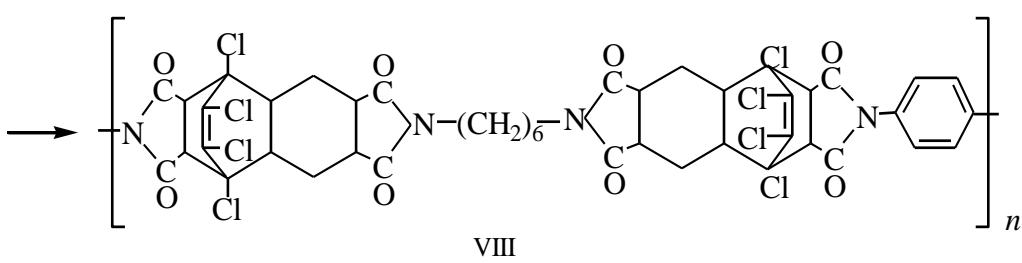
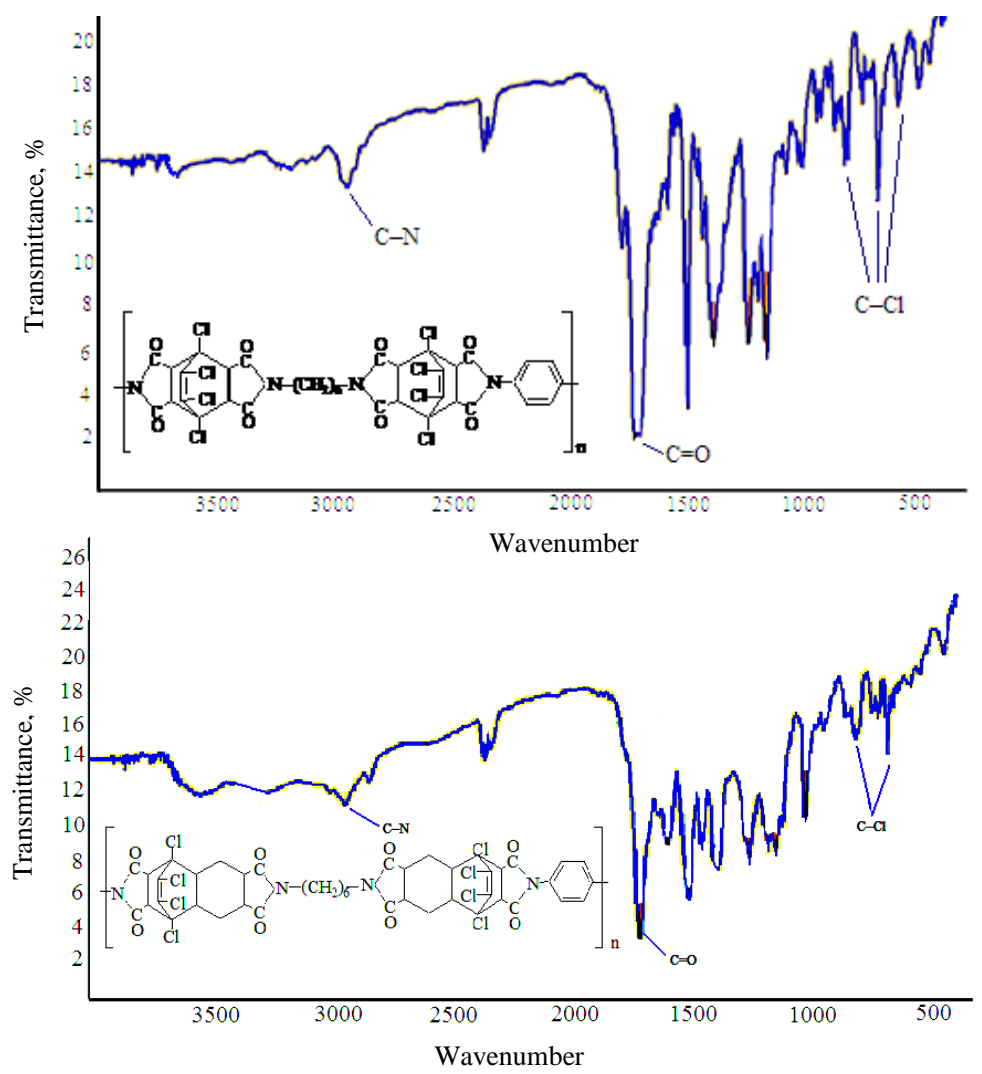

Fig.2. IR spectrum of polyimides VII and VIII. 
In the solutions of polyimides VII and VIII in determining [ $\eta$ ] the polyelectrolyte effect is not observed (Figure 3). Therefore, the molecular mass (MM) of the polyimides was judged by the reduced viscosity $\eta_{\mathrm{re}}$ of the reaction solution.

The investigations of the influence of the monomers concentration on $\eta_{\text {re }}$ showed that $\eta_{\text {re }}$ polyimides based on diene-dienophilic bisimides (V $a, b, c)$ and (VI $a, b, c)$ depends on the bi- and tricyclic fragment and is a curve with a maximum of the corresponding initial mono-

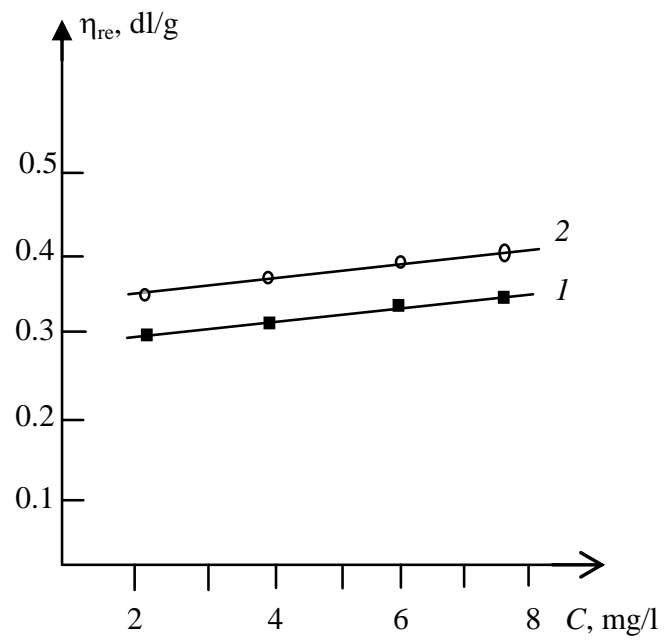

Fig. 3. Dependence of $\eta_{\mathrm{re}}$ on the concentration of polyimides on the basis of the diene-dienophiles: 1 - VII, 2 - VIII.

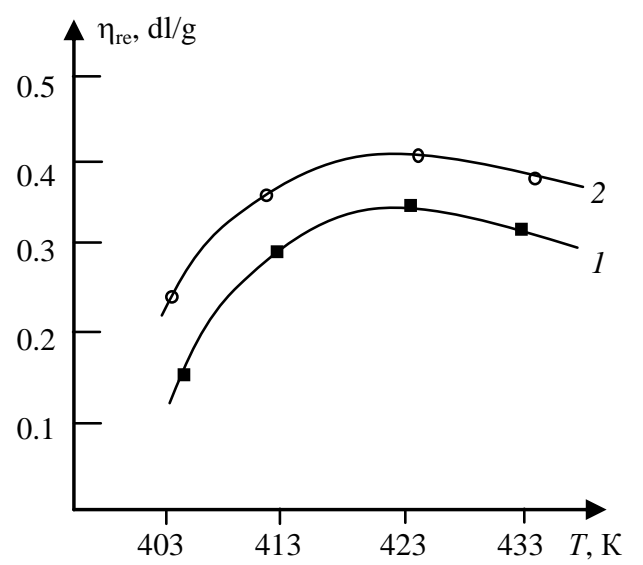

Fig. 5. Dependence of $\eta_{\text {re }}$ on temperature of polyimides on the basis of the diene-dienophiles: 1 - VII, 2 - VIII. mers (Figure 3). The optimum monomer concentration is 20 mas \%. Increasing the concentration above the optimum leads to a decrease in the MM polyimide.

The influence of the reaction temperature on the MM polyimide is shown in Figure 3, where $\eta_{\text {re }}$ increases with increasing temperature: at $130^{\circ} \mathrm{C} \eta_{\mathrm{re}} 0.10-0.15 \mathrm{dl} / \mathrm{g}$, at $140^{\circ} \mathrm{C} 0.22-0.26$ $\mathrm{dl} / \mathrm{g}$, at $150^{\circ} \mathrm{C} \quad 0.36-0.38 \mathrm{dl} / \mathrm{g}$, respectively, temperature increase to $160^{\circ} \mathrm{C}$ leads to a decrease in $\eta_{\text {re }}$ to $0.30-0.25 \mathrm{dl} / \mathrm{g}$.

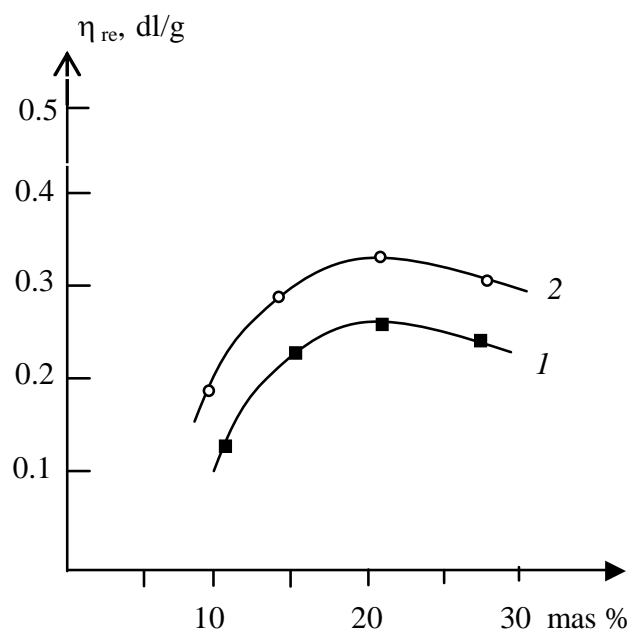

Fig. 4. Dependence of $\eta_{\text {re }}$ of $0.5 \%$ solution of polyimides on the basis of the diene-dienophiles: 1 - VII, 2 - VIII.

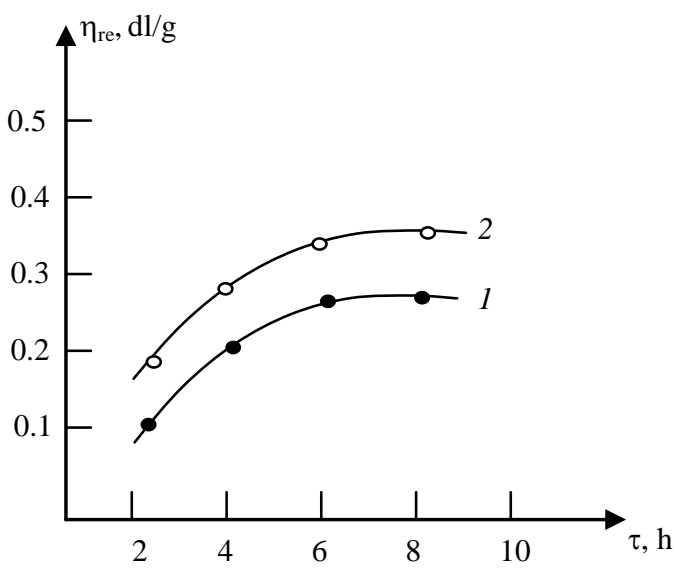

Fig. 6. Dependence of $\eta_{\text {re }}$ on of duration of polyimides on the basis of the diene-dienophiles: 1 - VII, 2 - VIII. 
Optimal duration of the reaction reacher at $150^{\circ} \mathrm{C}$ for $6-8$ hours. Increase the duration leads to a decrease in $\eta_{\mathrm{re}}$.

Thus, the study of the formation of polyimides VII and VIII on the basis of the dienedienophilic bisimides (V $b, c)$ and (VI $b, c)$ in DMA showed that the greatest value of $\eta_{\mathrm{re}}$ is achieved when the concentration of monomers in 20 mass $\%$ at $150^{\circ} \mathrm{C}$ for $6-8$ hours (Figures 5, $6)$. The yield of polyimides under these conditions is $90-95 \%$.

The obtained PI are well soluble in polar solvents (DMF, DMA, N-methylpyrrolidone, DMSO and pyridine). They are heat-resistant up to $300-360^{\circ} \mathrm{C}$. The loss in mass PI of this temperature is $5-6 \%$.

Hence our research demonstrated the possibility to obtain hinge-ladder polyimides with the desired spatial structure.

\section{Experimental part}

IR spectra of synthesized compounds were recorded on a spectrophotometer UR-20 from mulls in mineral oil [7]. UV spectrum were obtained on a spectrophotometer Specord UV-Vis from solutions in methanol, concentration $1.5 \times 10^{-4} \mathrm{~mol} \mathrm{l}^{-1}$. As internal reference we used $\mathrm{CCl}_{4}$. Molecular mass was determined by the method [7] of melting with camphor.

The purity of compounds was checked by TLC on Silufol UV-254 plates, eluent benzenedichloroethane-AcOH, 8:3:1, development under UV irradiation [8].

The reduced viscosity $\eta$ was measured using Ubbelohde viscometer with extrapolation of the values $\eta_{\mathrm{re} / c}\left(\right.$ at $25^{\circ} \mathrm{C}$ ) of the polymer solution in DMF to the zero concentration [9].

Anhydride of 1,4,5,6,7,7-hexachlorobicyclo[2.2.1] hept-5-ene-2,3-dicarboxylic acid was prepared by the procedure [10].

Anhydride of endo, exo-1,2,3,4,11,11-hexachlorotricyclo[6.2.1.0 $0^{5,10}$ undec-2-ene-7,8-dicarboxylic acid was prepared by the procedure [11].

N-Substituted cyclic imides of 1,4,5,6, 7,7hexachlorobicyclo[2.2.1]hept-5-ene-2,3-dicarboxylic acid (I $\boldsymbol{a}, \boldsymbol{b}, \boldsymbol{c})$. To a solution of $0.01 \mathrm{~mol}$ of 1,4,5,6,7,7-hexachlorobicyclo[2.2.1] hept-5-ene2,3-dicarboxylic acid anhydride in $50 \mathrm{ml}$ of
DMF was added at stirring $0.01 \mathrm{~mol}$ of an appropriate diamine in $10 \mathrm{ml}$ of DMF, the mixture was stirred for $6 \mathrm{~h}$ at $150^{\circ} \mathrm{C}$, cooled to room temperature, and poured in ice water. The precipitated crystals were filtered off, washed with water several times, dried, and analyzed.

$\mathrm{N}$-(2-Aminoethyl)imide of 1,4,5,6,7,7-hexachlorobicyclo[2.2.1]hept-5-ene-2,3-dicarboxylic acid (I $\boldsymbol{a}$ ). Yield $-91 \%$, m.p. $-133^{\circ} \mathrm{C}$ (benzene), $R_{f}-0.71$. IR spectrum, $v, \mathrm{~cm}^{-1}: 3350$ $\left(\mathrm{NH}_{2}\right), 1720,1780(\mathrm{C}=\mathrm{O}), 1601(\mathrm{C}=\mathrm{C}), 680-$ $750(\mathrm{C}-\mathrm{Cl})$. Found, \%: C 31.61, $\mathrm{H} 1.98, \mathrm{Cl}$ 36.58, N 7.70. $M$ 413. $\mathrm{C}_{11} \mathrm{H}_{8} \mathrm{Cl}_{6} \mathrm{~N}_{2} \mathrm{O}_{2}$. Calculated, \%: C 47.48, $\mathrm{H} \mathrm{2.96,} \mathrm{Cl} 34.80, \mathrm{~N} 4.58 . M$ 413.09.

$\mathrm{N}$-(6-Aminohexyl)imide of 1,4,5,6,7,7-hexachlorobicyclo[2.2.1] hept-5-ene-2,3-dicarboxylic acid (I b). Yield $-94 \%$, m.p. $-143^{\circ} \mathrm{C}$ (benzene), $R_{f}-0.68$. IR spectrum, $v, \mathrm{~cm}^{-1}: 3420$ $\left(\mathrm{NH}_{2}\right), 1780(\mathrm{C}=\mathrm{O}), 1606(\mathrm{C}=\mathrm{C}), 650-680(\mathrm{C}-$ $\mathrm{Cl})$. Found, \%: $\mathrm{C} 37.92, \mathrm{H} \mathrm{3.09,} \mathrm{Cl} 44.88, \mathrm{~N}$ 5.41. $M$ 468. $\mathrm{C}_{15} \mathrm{H}_{16} \mathrm{Cl}_{6} \mathrm{~N}_{2} \mathrm{O}_{2}$. Calculated, \%: $\mathrm{C}$ 38.41, H 3.43, Cl 45.35, N 5.97. M 468.99.

$\mathrm{N}$-(2-Aminohenyl)imide of 1,4,5,6,7,7-hexachlorobicyclo[2.2.1] hept-5-ene-2,3-dicarboxylic acid (I c). Yield $-92 \%$, m.p. $-145^{\circ} \mathrm{C}$ (petroleum ether), $R_{f}-0.67$. IR spectrum, $v, \mathrm{~cm}^{-1}$ : $3350\left(\mathrm{NH}_{2}\right), 1780(\mathrm{C}=\mathrm{O}), 1601(\mathrm{C}=\mathrm{C}), 650-$ $710(\mathrm{C}-\mathrm{Cl})$. Found, \%: C 40.82, $\mathrm{H} 1.81, \mathrm{Cl}$ 49.06, N 5.41. $M$ 460. $\mathrm{C}_{15} \mathrm{H}_{8} \mathrm{Cl}_{6} \mathrm{~N}_{2} \mathrm{O}_{2}$. Calculated, \%: C 39.04, $\mathrm{H} \mathrm{1.76,} \mathrm{Cl} 44.05, \mathrm{~N}$ 6.07. $M$ 480.92 .

N-Substituted cyclic imides of endo, exo1,2,3,4,11,11-hexachlorotricyclo-[6.2.1.0 $\left.{ }^{5,10}\right]$ undec-2-ene-7,8-dicarboxylic acid (II $a, b, c$ ). Similarly from $0.01 \mathrm{~mol}$ of endo, exo-1,2,3,4,11,11-hexachlorotricyclo[6.2.1.0, $\left.0^{5,10}\right]$ undec-2-ene-7,8-dicarboxylic acid anhydride and $0.01 \mathrm{~mol}$ of an appropriate diamine aminoimide II $a, b, c$ was prepared.

N-(2-Aminoethyl)imide of endo, exo-1,2, 3,4,11,11-hexachlorotricyclo-[6.2.1.0 $\left.{ }^{5,10}\right]$ undec2-ene-7,8-dicarboxylic acid (II $\boldsymbol{a}$ ). Yield - 92\%, m.p. $-120^{\circ} \mathrm{C}$ (benzene), $R_{f}-0.70$. IR spectrum, $v, \mathrm{~cm}^{-1}: 3280\left(\mathrm{NH}_{2}\right), 1720,1780(\mathrm{C}=\mathrm{O}), 1601$ $(\mathrm{C}=\mathrm{C}), 600-705(\mathrm{C}-\mathrm{Cl})$. Found, \%: $\mathrm{C} 43.86, \mathrm{H}$ 2.81, $\mathrm{Cl}$ 41.01, N 6.01. $M$ 466. $\mathrm{C}_{15} \mathrm{H}_{14} \mathrm{Cl}_{6} \mathrm{~N}_{2} \mathrm{O}_{2}$. 
Calculated, \%: C 39.58, H 3.00, $\mathrm{Cl} 47.30, \mathrm{~N}$ 6.00. $M 467$.

$\mathrm{N}$-(6-Aminohexyl)imide of endo, exo-1,2, 3,4,11,11-hexachlorotricyclo[6.2.1.0 $\left.{ }^{5,10}\right]$ undec2-ene-7,8-dicarboxylic acid (II b). Yield - 95\%, m.p. $153^{\circ} \mathrm{C}$ (benzene), $R_{f}-0.69$. IR spectrum, $v, \mathrm{~cm}^{-1}: 3280,3420\left(\mathrm{NH}_{2}\right), 1720,1780(\mathrm{C}=\mathrm{O})$, $1601(\mathrm{C}=\mathrm{C}), 602-710(\mathrm{C}-\mathrm{Cl})$. Found, \%: $\mathrm{C}$ 42.88, $\mathrm{H}$ 3.96, $\mathrm{Cl}$ 40.21, $\mathrm{N}$ 5.01. M 522. $\mathrm{C}_{19} \mathrm{H}_{22} \mathrm{Cl}_{6} \mathrm{~N}_{2} \mathrm{O}_{2}$. Calculated, \%: $\mathrm{C}$ 43.62, $\mathrm{H}$ 4.23, $\mathrm{Cl}$ 40.66, N 5.35. M 523.07.

$\mathrm{N}$-(2-Aminohenyl)imide of endo, exo-1,2, 3,4,11,11-hexachlorotricyclo[6.2.1.0 $\left.{ }^{5,10}\right]$ undec2-ene-7,8-dicarboxylic acid (II c). Yield - 94\%, m.p. $-121^{0} \mathrm{C}$ (benzene), $R_{f}-0.68$. IR spectrum, $v, \mathrm{~cm}^{-1}: 3280\left(\mathrm{NH}_{2}\right), 1720,1780(\mathrm{C}=\mathrm{O}), 1606$ $(\mathrm{C}=\mathrm{C}), 600-702(\mathrm{C}-\mathrm{Cl})$. Found, \%: $\mathrm{C} 43.86, \mathrm{H}$ 2.30, $\mathrm{Cl}$ 40.91, N 5.01. $M$ 514. $\mathrm{C}_{19} \mathrm{H}_{14} \mathrm{Cl}_{6} \mathrm{~N}_{2} \mathrm{O}_{2}$. Calculated, \%: C 44.31, $\mathrm{H} 2.73, \mathrm{Cl} 41.30, \mathrm{~N}$ 5.43. $M$ 515.01.

Maleinimidoalkyl(aryl)substituted imides of 1,4,5,6,7,7-hexachlorobicycle[2.2.1] hept5-ene-2,3-dicarboxylic acid (III $\boldsymbol{a}, \boldsymbol{b}, \boldsymbol{c}$ ). To a solution of $0.05 \mathrm{~mol}$ of aminoimide (I $a, b, c)$ in $50 \mathrm{ml}$ of DMF was added $0.05 \mathrm{~mol}$ of maleic anhydride, the mixture was stirred for $6 \mathrm{~h}$ at $150^{\circ} \mathrm{C}$, cooled, and poured in ice water. The precipitate was filtered off, washed with water, and dried.

$\mathrm{N}$-[2-Maleimido)ethyl]imide of $1,4,5,6,7$, 7-hexachlorobicyclo[2.2.1] hept-5-ene-2,3-dicarboxylic acid III a. Yield $-85 \%$, m.p. $-133^{\circ} \mathrm{C}$ (benzene-hexane), $\mathrm{R}_{\mathrm{f}}-0.67$. IR spectrum, $v, \mathrm{~cm}^{-1}$ : $1710,1780(\mathrm{C}=\mathrm{O}), 1600(\mathrm{C}=\mathrm{C}), 680-800(\mathrm{C}-\mathrm{Cl})$. Found, \%: C 38.35, H 1.28, Cl 42.91, N 5.01. $M$ 462. $\mathrm{C}_{15} \mathrm{H}_{8} \mathrm{Cl}_{6} \mathrm{~N}_{2} \mathrm{O}_{4}$. Calculated, \%: $\mathrm{C} 44.10, \mathrm{H}$ 1.63, $\mathrm{Cl} 41.10, \mathrm{~N}$ 5.60. M 463.93.

N-[6-Maleimido)hexyl]imide of $1,4,5,6$, 7,7-hexachlorobicyclo[2.2.1] hept-5-ene-2,3-dicarboxylic acid III b. Yield $-99 \%$, m.p. $-123^{\circ} \mathrm{C}$ (benzene-hexane), $\mathrm{R}_{\mathrm{f}}-0.46$. IR spectrum, $\mathrm{v}$, $\mathrm{cm}^{-1}: 1840(\mathrm{C}=\mathrm{O}), 1590-1600(\mathrm{C}=\mathrm{C}), 650-720$ (C-Cl). Found, \%: C 48.91, H 2.14, Cl 33.41, N 4.21. $M$ 640. $\mathrm{C}_{19} \mathrm{H}_{16} \mathrm{Cl}_{6} \mathrm{~N}_{2} \mathrm{O}_{4}$. Calculated, \%: $\mathrm{C}$ 49.32, H 2.53, Cl 33.59, N 4.42. M 641.06.

$\mathrm{N}$-[4-Maleimido)phenyl]imide of $1,4,5,6$, 7,7-hexachlorobicyclo[2.2.1] hept-5-ene-2,3-dicarboxylic acid (III c). Yield - 95\%, m.p. - $181^{\circ} \mathrm{C}$ (benzene-hexane), $R_{f}-0.68$. IR spectrum, $v, \mathrm{~cm}^{-1}: 1710,1780(\mathrm{C}=\mathrm{O}), 1600-1610$ $(\mathrm{C}=\mathrm{C}), 680-750(\mathrm{C}-\mathrm{Cl})$. Found, \%: $\mathrm{C} 42.01, \mathrm{H}$ 1.04, $\mathrm{Cl}$ 39.12, N 4.84. $M$ 530. $\mathrm{C}_{19} \mathrm{H}_{8} \mathrm{Cl}_{6} \mathrm{~N}_{2} \mathrm{O}_{4}$. Calculated, \%: C 42.26, H 1.49, Cl 39.39, N 5.12. M 529 .

Maleinimidoalkyl(aryl) substituted imides of endo, exo-1,2,3,4,11,11-hexachlorotricyclo-[6.2.1.0 $\left.{ }^{5,10}\right]$ undec-2-ene-7,8-dicarboxylic acid (IV $\boldsymbol{a}, \boldsymbol{b}, \boldsymbol{c})$. In the same way as above from $0.05 \mathrm{~mol}$ of aminoimide of endo, exo-1,2, $3,4,11,11$-hexachlorotricyclo[6.2.1. $\left.0^{5,10}\right]$ undec2-ene-7,8-dicarboxylic acid II a, b, c and maleic anhydride were obtained bisimide IV $a, b, c$.

$\mathrm{N}$-[2-Maleimido)ethyl]imide of endo, exo1,2,3,4,11,11-hexachlorotricyclo[6.2.1.0 $\left.{ }^{5,10}\right]$-undec-2-ene-7,8-dicarboxylic acid (IV $a$ ). Yield $92 \%$, m.p. $-400^{\circ} \mathrm{C}\left(\mathrm{CHCl}_{3}\right), R_{f}-0.68$. IR spectrum, $v, \mathrm{~cm}^{-1}: 1710,1780(\mathrm{C}=\mathrm{O}), 1610(\mathrm{C}=\mathrm{C})$, 670-750 (C-Cl). Found, \%: C 40.01, H 2.00, Cl 36.86, N 4.92. $M$ 530. $\mathrm{C}_{19} \mathrm{H}_{14} \mathrm{Cl}_{6} \mathrm{~N}_{2} \mathrm{O}_{4}$. Calculated, \%: C 42.93, H 2.43, Cl 40.26, N 4.85 . M 531 .

N-[6-Maleimido)hexyl]imide of endo, exo-1,2,3,4,11,11-hexachlorotricyclo[6.2.1.0 $\left.{ }^{5,10}\right]$ undec-2-ene-7,8-dicarboxylic acid (IV b). Yield $-90 \%$, m.p. $-270^{\circ} \mathrm{C}\left(\mathrm{CHCl}_{3}\right), R_{f}-0.70$. IR spectrum, $v, \mathrm{~cm}^{-1}$ : 1710, $1780(\mathrm{C}=\mathrm{O}), 1610(\mathrm{C}=\mathrm{C})$, 670-750 (C-Cl). Found, \%: C 50.67, H 4.10, Cl 24.89, N 5.02. $M 581.60 \mathrm{C}_{23} \mathrm{H}_{22} \mathrm{Cl}_{6} \mathrm{~N}_{2} \mathrm{O}_{4}$. Calculated, \%: $\mathrm{C}$ 47.01, $\mathrm{H} 3.74, \mathrm{Cl} 26.4, \mathrm{~N} 5.25$. M 582.21.

$\mathrm{N}$-[4-Maleimido)phenyl]imide of endo, exo-1,2,3,4,11,11-hexachlorotricyclo[6.2.1.0 $\left.{ }^{5,10}\right]$ undec-2-ene-7,8-dicarboxylic acid (IV c). Yield $-91 \%$, m.p. $-120^{\circ} \mathrm{C}$ (methanol), $R_{f}-0.68$. IR spectrum, v, $\mathrm{cm}^{-1}$ : 1710, $1780(\mathrm{C}=\mathrm{O}), 1610$ $(\mathrm{C}=\mathrm{C}), 670-750(\mathrm{C}-\mathrm{Cl})$. Found, \%: C 52.90, $\mathrm{H}$ 4.10, $\mathrm{Cl}$ 24.89, N 5.02. $M$ 576.0. $\mathrm{C}_{23} \mathrm{H}_{14} \mathrm{Cl}_{6} \mathrm{~N}_{2} \mathrm{O}_{4}$. Calculated, \%: C 47.60, H 3.74, Cl 26.4, N 5.25. M 579.15.

Maleinimidoalkyl(aryl) substituted imides of 1,2,3,4-tetrachlorocyclohexa-1,3-diene5,6-dicarboxylic acid ( $\mathrm{V} \boldsymbol{a}, \boldsymbol{b}, \boldsymbol{c}) .0 .01 \mathrm{~mol}$ of 1,4,5,6,7,7-hexachlorobicyclo[2.2.1]hept-5-ene2,3-dicarboxylic acid imide (III $a, b, c$ ) and 50 $\mathrm{ml}$ of pyridine was added while stirring at room temperature to $200 \mathrm{ml}$ of $\mathrm{DMF}$. The reaction 
proceeded with heat evolution and self-heated to $50^{\circ} \mathrm{C}$. Then the mixture was stirred again for $2 \mathrm{~h}$ at $110-120^{\circ} \mathrm{C}$. On cooling the mixture was poured in ice water. The precipitate was filtered off, washed with water, and recrystallized from methanol.

$\mathrm{N}$-[2-Maleimido)ethyl]imide of $1,2,3,4-$ tetrachlorocyclohexa-1,3-diene-5,6-dicarboxylic acid (V a). Yield $-79 \%$, m.p. $-146-147^{0} \mathrm{C}$. $R_{f}-0.66$. IR spectrum, $v, \mathrm{~cm}^{-1}: 1700,1780$ $(\mathrm{C}=\mathrm{O}), 1605(\mathrm{C}=\mathrm{C}), 670-760(\mathrm{C}-\mathrm{Cl})$. Found, \%: C 40.51, H 1.90, Cl 31.09, N 6.72. M 410. $\mathrm{C}_{14} \mathrm{H}_{8} \mathrm{Cl}_{4} \mathrm{~N}_{2} \mathrm{O}_{4}$. Calculated, \%: C 40.97, H 1.93, Cl 34.50, N 6.81. M 411.01.

$\mathrm{N}$-[6-Maleimido)hexyl]imide of $1,2,3,4$ tetrachlorocyclohexa-1,3-diene-5,6-dicarboxylic acid (V b). Yield $-75 \%$, m.p. $-134-135^{\circ} \mathrm{C}$, $R_{f}-0.58$. IR spectrum, $v, \mathrm{~cm}^{-1}: 1710,1780$ $(\mathrm{C}=\mathrm{O}), 1610(\mathrm{C}=\mathrm{C}), 670-750(\mathrm{C}-\mathrm{Cl})$. Found, \%: C 46.18, H 3.41, Cl 30.01, N 6.02. M 466. $\mathrm{C}_{18} \mathrm{H}_{16} \mathrm{Cl}_{4} \mathrm{~N}_{2} \mathrm{O}_{4}$. Calculated, \%: C 46.38, $\mathrm{H}$ 3.44, Cl 30.42, N 6.00. $M 466.11$.

$\mathrm{N}$-[4-Maleimido)phenyl]imide of 1,2,3,4tetrachlorocyclohexa-1,3-diene-5,6-dicarboxylic acid (V c). Yield $-90 \%$, m.p. $-328-330^{\circ} \mathrm{C}$, $R_{f}-0.66$. IR spectrum, $v, \mathrm{~cm}^{-1}: 1715,1780$ $(\mathrm{C}=\mathrm{O}), 1610(\mathrm{C}=\mathrm{C}), 670-750(\mathrm{C}-\mathrm{Cl})$. Found, \%: C 52.89, H 2.89, Cl 34.25, N 6.32. M 402. $\mathrm{C}_{18} \mathrm{H}_{14} \mathrm{Cl}_{4} \mathrm{~N}_{2} \mathrm{O}_{4}$. Calculated, \%: C 53.50, $\mathrm{H}$ 3.48, Cl 34.80, N 6.93. $M$ 403.99.

Maleinimidoalkyl(aryl)substituted imides of endo, exo-2,3,4,5-tetrachlorobicyclo [4. 4.0]deca-2,4-diene-8,9-dicarboxylic acid (VI $a$, $\boldsymbol{b}, \boldsymbol{c})$ were similarly obtained from bisimide of endo, exo-1,2,3,4,11,11-hexachlorotricyclo[6.2.1.0 $\left.0^{5,10}\right]$ undec-2-ene-7,8-dicarboxylic acid (IV $a, b, c)$.

N-[2-Maleimido)ethyl]imide of endo, exo-2,3,4,5-tetrachlorobicyclo[4.4.0]deca-2,4diene-8,9-dicarboxylic acid (VI $a$ ). Yield $88 \%$, m.p. $-148^{0} \mathrm{C} . R_{f}-0.6$. IR spectrum, $v$, $\mathrm{cm}^{-1}: 1715,1780(\mathrm{C}=\mathrm{O}), 1605(\mathrm{C}=\mathrm{C}), 670-760$ (C-Cl). Found, \%: C 46.11, H 2.88, Cl 30.02, N 5.92. $M$ 464. $\mathrm{C}_{18} \mathrm{H}_{14} \mathrm{Cl}_{4} \mathrm{~N}_{2} \mathrm{O}_{4}$. Calculated, \%: C 46.78, H 3.03, Cl 30.55, N 6.03. M 464.09.

$\mathrm{N}$-[6-Maleimido)hexyl]imide of endo, exo-2,3,4,5-tetrachlorobicyclo[4.4.0]deca-2,4diene-8,9-dicarboxylic acid (VI $b$ ). Yield $90 \%$, m.p. $-152^{\circ} \mathrm{C}, R_{f}-0.58$. IR spectrum, v, $\mathrm{cm}^{-1}: 1720,1780(\mathrm{C}=\mathrm{O}), 1610(\mathrm{C}=\mathrm{C}), 670-750$ (C-Cl). Found, \%: C 50.43, H 3.91, Cl 26.89, N 4.88. M 519.28. $\mathrm{C}_{22} \mathrm{H}_{22} \mathrm{Cl}_{4} \mathrm{~N}_{2} \mathrm{O}_{4}$. Calculated, \%: C 50.78, H 4.24, Cl 27.26, N 5.38. M 520.20.

$\mathrm{N}$-[4-Maleimido)phenyl]imide of endo, exo-2,3,4,5-tetrachlorobicyclo[4.4.0]deca-2,4diene-8,9-dicarboxylic acid (VI c). Yield $92 \%$, m.p. $-166^{\circ} \mathrm{C}, R_{f}-0.62$. IR spectrum, v, $\mathrm{cm}^{-1}: 1720,1780(\mathrm{C}=\mathrm{O}), 1610(\mathrm{C}=\mathrm{C}), 670-750$ (C-Cl). Found, \%: C 52.89, H 2.89, Cl 34.25, N 6.32. $M$ 512. $\mathrm{C}_{22} \mathrm{H}_{14} \mathrm{Cl}_{4} \mathrm{~N}_{2} \mathrm{O}_{4}$. Calculated, \%: C 51.60, H 2.75, Cl 27.70, N 5.46. M 512.14.

Polyimides VII on the basis of N-[6-maleimido)hexyl]imide- ( $\mathrm{V} b$ ) and N-[4-maleimido)-phenyl]imide of 1,2,3,4-tetrachlorocyclohexa-1,3-diene-5,6-dicarboxylic acid (V c ). A mixture of $0.05 \mathrm{~mol}$ of bisimide $(\mathrm{V} b),(\mathrm{V} c)$ and $0.05 \mathrm{~g}$ of hydroquinonein $20 \mathrm{ml}$ of anhydrous DMA was stirred for $6 \mathrm{~h}$ at $140^{\circ} \mathrm{C}$. On cooling the reaction mixture was poured into ice water. The precipitated crystals were filtered off, washed with water, dried, and recrystallized from methanol. Yield - 92\%, m.p. - 304$315^{\circ} \mathrm{C}, R_{f}-0.71$. Intrinsic viscosity (DMF, $\left.25^{\circ} \mathrm{C}\right) \eta 0.22 \mathrm{dl} / \mathrm{g}$. IR spectrum, $v, \mathrm{~cm}^{-1}: 1710$, $1780(\mathrm{C}=\mathrm{O}), 1610(\mathrm{C}=\mathrm{C}), 680-750(\mathrm{C}-\mathrm{Cl})$. Found, \%: C 54.28, H 2.01, N 4.96, Cl 25.62. $\mathrm{C}_{25} \mathrm{H}_{14} \mathrm{Cl}_{4} \mathrm{~N}_{2} \mathrm{O}_{4}$. Calculated, \%: C 54.77, $\mathrm{H}$ 2.57, N 5.10, Cl 25.80.

Polyimides VIII on the basis of N-[6maleimido)hexyl]imide- (VI b) and N-[4-maleimido)phenyl]imide of endo, exo-2,3,4,5-tetrachlorobicyclo[4.4.0]deca-2,4-diene-8,9-dicarboxylic acid (VI $c$ ) was similarly obtained from bisimides (VI $\boldsymbol{b}, \boldsymbol{c}$ ). Yield - 94\%, m.p. - 328$330^{\circ} \mathrm{C}, R_{f}-0.75, \eta-0.28 \mathrm{dl} / \mathrm{g}$. IR spectrum, $v$, $\mathrm{cm}^{-1}: 1712,1780(\mathrm{C}=\mathrm{O}), 1608(\mathrm{C}=\mathrm{C}), 600-750$ $(\mathrm{C}-\mathrm{Cl})$. Found, \%: C 57.28, $\mathrm{H} 3.01, \mathrm{~N} 4.21, \mathrm{Cl}$ 22.98. $\mathrm{C}_{29} \mathrm{H}_{20} \mathrm{Cl}_{4} \mathrm{~N}_{2} \mathrm{O}_{4}$. Calculated, \%: C 57.83, H 3.34, N 4.65, Cl 23.54.

\section{References}

1. Bessonov M.N., Koton M.M., Kudriavtcev V.V., Laius L.A. Poliimidy - class termostoikikh polimerov. M.: Nauka, 1983. 328 s.

2. Vinogradova S.V., Vasnev V.A. Polikondensatcionnye protcessyi polimery. M.: Nauka, 2000. $250 \mathrm{~s}$.

3. Korshak V.V., Rusanov A.L., Batirov I.G. Novoe v oblasti termostoikikh poliimidov. Dushanbe: Donish, 1986. 102 s. 
4. Mamedov S.A., Kuliev A.B., Akhmedov T.G., Zefirov N.S. Irratcionalnyi sintez 1,2,3,4-tetrakhlor1,3-tciclogeksadien-5,6-dikarbonovoi kisloty // Zhurn. org. himii. 1991. T. 27. № 10. S. 2228 2229.

5. Bublik L.S., Moiseev V.D., Chernova A.G., Piliaeva V.F., Nekrasova L.N. Vliianie rastvoritelia na protcess termicheskoi imidizatcii poliamidokislot // Plastmassy. 1974. № 3. S. 10-13.

6. Arhipova I.A., Zhubanov B.A., Rafikov S.R. Novye geterotciclicheskie polimery na osnove tciclicheskikh imidov karbonovykh kislot // Uspehi himii. 1978. T. 47. № 4. S. 705-738.

7. Nakanisi K. Infrakrasnye spektryi stroenie organicheskikh soedinenii. M.: Mir, 1965. 209 s.
8. Akhrem A.A., Kuznetcov A.I. Tonkosloinaia khromatografiia. M.: Nauka, 1964. $175 \mathrm{~s}$.

9. Rafikov S.R., Pavlova S.A., Tverdokhlebova I.I. Metody opredeleniia molekuliarnykh vesov i polidispersnosti vysokomolekuliarnykh soedinenii. M.: Izd-vo AN SSSR, 1963. 334 s.

10. Simonov V.D., Antonov L.T., Nedelchenko B.M., Scliar S.Ia. Angidrid 1,4,5,6,7,7-geksakhlorbitciclo[2.2.1]gept-5-en-2,3-dikarbonovoikisloty. A.s. 363079 SSSR. B.I. 1973. № 8.

11. Salahov M.S., Guseinov M.M., Kiazimova T.G., Treivus E.M., Alekperov N.A. Sposob polucheniia angidrida 1,2,3,4,11,11-geksakhlortritciklo[2.4. 1.0]undetc-2-dikarbonovoi kisloty. A.s. 172757 SSSR. B.I. 1965. № 14 .

\title{
DİEN-DİENOFIL FRAQMENTLİ BİSIMMIDLəR OSASINDA DILS-ALDER REAKSIYYASI ÜZRə POLIXLORLU TSIKLIK DİKARBON TURŞULARININ POLIIIMIDLORININ SINTEZI
}

\author{
A.İ.Đlixanova \\ Polixlorlu tsiklik dikarbon turşularının $\mathrm{N}$-aminoimidlərinin sintezi öyrənilmiş və onlar əsasında Dils-Alder reaksiyası \\ üzrə hərəki-nərdivan tipli poliimiidlərin alınmasının mümkünlüyü göstərilmişdir. \\ Açar sözlor: Dils-Alder reaksiyası, bisimidlər, poliimidlar, asillaşmə, bisimidodienlər.

\section{СИНТЕЗ ПОЛИИМИДОВ ПОЛИХЛОРИРОВАННЫХ ЦИКЛИЧЕСКИХ ДИКАРБОНОВЫХ КИСЛОТ НА ОСНОВЕ ДИЕН-ДИЕНОФИЛЬНЫХ БИСИМИДОВ ПО РЕАКЦИИ ДИЛЬСА-АЛЬДЕРА}

\section{А.И.Алиханова}

Изучены условия синтеза $\mathrm{N}$-аминоимидов полихлорированных циклических дикарбоновых кислот и показана возможность на их основе получения шарнирно-лестничных полиимидов по реакции Дильса-Альдера.

Ключевье слова: реакция Дильса-Альдера, бисимиды, полиимиды, ацилирование, бисимидодиеньл. 